\title{
AN ITERATIVE METHOD OF GENERAL PLANETARY THEORY
}

\author{
V. A. BRUMBERG \\ Institute of Theoretical Astronomy, Leningrad, U.S.S.R.
}

\begin{abstract}
This paper deals with an iterative version of the general planetary theory. Just as in Airy's Lunar method the series in powers of planetary masses are replaced here by the iterations to achieve improved approximations for the coefficients of planetary inequalities. The right-hand members of the equations of motion are calculated in closed formulas, and no expansion in powers of small corrections to the planetary coordinates is needed. For the $N$-planet case this method requires the performance of the analytical operations on a computer with power series of $4 N$ polynomial variables, the coefficients being the exponential series of $N-1$ angular arguments. To obtain numerical series of planetary motion one has to solve the secular system using Birkhoff's normalization or the Taylor series in powers of time. A slight modification of the method in the resonant case makes it valid for the treatment of the main problem of the Galilean satellites of Jupiter.
\end{abstract}

\section{Introduction}

This paper deals with an iterative version of the general planetary theory proposed by the author in 1969 (Brumberg, 1970). The general method is based on the ideas by Hill (non-Keplerian intermediary), von Zeipel (separation of slow and fast variables) and Birkhoff (reduction of dynamical system to normal form). As in all existing planetary theories the actual performance of this method involves the expansions in powers of planetary masses. Such a method was used in the second-order linear theory of secular perturbations (Brumberg and Egorova, 1971) and in the general first-order theory (Brumberg and Chapront, 1974).

Expansions in powers of planetary masses are convenient in construction of first-order theory permitting us to consider separately the perturbations in motion of planet $i$ due to planet $j$. In second-order theory in addition to these single perturbations one has to deal with double perturbations caused by the combined action of planets $j$ and $k$. In third-order theory triple perturbations arise from the combined influence of planets $j, k$ and $l$, etc. Expressions of these perturbations become rather cumbersome and expansions in powers of planetary masses lose their efficiency. That is why these expansions are not used here. Dependence of masses is taken into account numerically by means of iterations. The only criterion of smallness of any term is its numerical value. This approach is similar to the Airy method used in the modern theory of the Moon. It should be noted that iterative versions of some classic methods of planetary perturbations have been already successfully elaborated (Broucke, 1969; Seidelmann, 1970). In the version suggested here we apply a new algorithm for computation of the right members. This algorithm does not demand expansions in powers of unknown variables.

Construction of the general planetary theory by this iterative method calls for a system of literal manipulation on computer with power series of $4 \mathrm{~N}$ polynomial variables, the coefficients being the exponential series of $N-1$ angular arguments 
(to be more precise, we have $N$ arguments but the sum of indices is always zero). Solution of this problem for $\mathrm{N}=9$ is possible on modern computers.

\section{Intermediary}

The general theory has been given in detail by Brumberg and Chapront (1974). We give here only the computational algorithm of the iterative version.

If $p_{i}$ and $w_{i}$ are related with heliocentric coordinates $x_{i}, y_{i}$ and $z_{i}$ by means of

$$
\begin{aligned}
& x_{i}+\sqrt{ }-1 y_{i}=a_{i}\left(1-p_{i}\right) \exp \sqrt{ }-1 \lambda_{i}, \quad z_{i}=a_{i} w_{i}, \\
& \lambda_{i}=n_{i} t+\varepsilon_{i}, \quad n_{i}^{2} a_{i}^{3}=k^{2}\left(m_{0}+m_{i}\right),
\end{aligned}
$$

then the equations of motion take the form

$$
\begin{aligned}
& \ddot{p}_{i}+2 \sqrt{ }-1 n_{i} \dot{p}_{i}-\frac{3}{2} n_{i}^{2}\left(p_{i}+q_{i}\right)=n_{i}^{2} P_{i}, \\
& \ddot{w}_{i}+n_{i}^{2} w_{i}=n_{i}^{2} W_{i} .
\end{aligned}
$$

Here

$$
\begin{aligned}
P_{i}= & -1-\frac{1}{2} p_{i}-\frac{3}{2} q_{i}+\left(1-p_{i}\right)\left(\frac{a_{i}}{r_{i}}\right)^{3}+\mu \sum_{j=1}^{N} \kappa_{i j}\left\{\zeta_{i j}^{-1}\left(\frac{a_{i}}{a_{j}}\right)^{2}\left(1-p_{j}\right)\left(\frac{a_{j}}{r_{j}}\right)^{3}+\right. \\
& \left.+\left(\frac{a_{i}}{a_{i j}}\right)^{2}\left[\frac{a_{i}}{a_{i j}}\left(1-p_{i}\right)-\frac{a_{j}}{a_{i j}}\left(1-p_{j}\right) \zeta_{i j}^{-1}\right]\left(\frac{a_{i j}}{\Delta_{i j}}\right)^{3}\right\} \\
W_{i}= & w_{i}-w_{i}\left(\frac{a_{i}}{r_{i}}\right)^{3}-\mu \sum_{j=1}^{N} \kappa_{i j}\left[\left(\frac{a_{i}}{a_{j}}\right)^{2} w_{j}\left(\frac{a_{j}}{r_{j}}\right)^{3}+\right. \\
& \left.+\left(\frac{a_{i}}{a_{i j}}\right)^{2}\left(\frac{a_{i}}{a_{i j}} w_{i}-\frac{a_{j}}{a_{i j}} w_{j}\right)\left(\frac{a_{i j}}{\Delta_{i j}}\right)^{3}\right]
\end{aligned}
$$

where

$$
\begin{aligned}
\mu \kappa_{i j}= & \frac{m_{j}}{m_{0}+m_{i}}, \quad \zeta_{i j}=\exp \sqrt{ }-1\left(\lambda_{i}-\lambda_{j}\right), \quad a_{i j}=\max \left(a_{i}, a_{j}\right), \\
\left(\frac{r_{i}}{a_{i}}\right)^{2}= & \left(1-p_{i}\right)\left(1-q_{i}\right)+w_{i}^{2}, \\
\left(\frac{\Delta_{i j}}{a_{i j}}\right)^{2}= & {\left[\frac{a_{i}}{a_{i j}}\left(1-p_{i}\right)-\frac{a_{j}}{a_{i j}}\left(1-p_{j}\right) \zeta_{i j}^{-1}\right]\left[\frac{a_{i}}{a_{i j}}\left(1-q_{i}\right)-\frac{a_{j}}{a_{i j}}\left(1-q_{j}\right) \zeta_{i j}\right]+} \\
& +\left(\frac{a_{i}}{a_{i j}} w_{i}-\frac{a_{j}}{a_{i j}} w_{j}\right)^{2},
\end{aligned}
$$

and $q_{i}=\bar{p}_{i}$. The form of expressions (2) and (3) is simpler for $a_{i j}=a_{i}$, but the choice made here is more suitable for computation.

We take as intermediary the particular solution of Equations (1), $p_{i}=p_{i}^{(0)}$ and $w_{i}=0$, containing $2 N$ arbitrary constants (mean motions $n_{i}$ and mean longitudes at 
the epoch $\varepsilon_{i}$ ). The planetary masses $m_{i}$ and mean motions $n_{i}$ are supposed to have fixed values which lead by Kepler's third law to definite values of the semimajor axes $a_{i}$. The intermediate solution can be represented in the exponential series form

$$
p_{i}^{(0)}=\sum p_{\gamma}^{(i)} \exp \sqrt{ }-1(\gamma \lambda)
$$

where

$$
(\gamma \lambda)=\gamma_{1} \lambda_{1}+\gamma_{2} \lambda_{2}+\cdots+\gamma_{N} \lambda_{N}
$$

and

$$
\gamma_{1}+\gamma_{2}+\cdots+\gamma_{N}=0
$$

Summation in (6) is extended over all integer values of $\gamma_{1}, \ldots, \gamma_{N}$. Coefficients $p_{\gamma}^{(i)}$ have real values which are determined by iterations. To do this we substitute expressions (6) known at some stage of the approximation process into right members (2). The pertinent series $P_{i}$ are of the same form (6)-(8) with coefficients $P_{\gamma}^{(i)}$. Improved values of the coefficients of (6) are then obtained as

$$
\begin{aligned}
& p_{0}^{(i)}=-\frac{1}{3} P_{0}^{(i)}, \\
& p_{\gamma}^{(i)}=n_{i}^{2} \frac{\left[(\gamma n)^{2}-2 n_{i}(\gamma n)+\frac{3}{2} n_{i}^{2}\right] P_{\gamma}^{(i)}-\frac{3}{2} n_{i}^{2} P_{-\gamma}^{(i)}}{(\gamma n)^{2}\left[n_{i}^{2}-(\gamma n)^{2}\right]}
\end{aligned}
$$

where $(\gamma n)$ means the dot product of $\gamma=\left(\gamma_{1}, \ldots, \gamma_{N}\right)$ and $n=\left(n_{1}, \ldots, n_{N}\right)$. The initial values of this process are $p_{i}^{(0)}=0$. Having found, with use of $(6)$ the exponential series,

$$
Z_{i}(k, l)=\left(1-p_{i}\right)^{k}\left(1-q_{i}\right)^{l}
$$

and

$$
Z_{i j}(k, l)=\left[\frac{a_{i}}{a_{i j}}\left(1-p_{i}\right)-\frac{a_{j}}{a_{i j}}\left(1-p_{j}\right) \zeta_{i j}^{-1}\right]^{k}\left[\frac{a_{i}}{a_{i j}}\left(1-q_{i}\right)-\frac{a_{j}}{a_{i j}}\left(1-q_{j}\right) \zeta_{i j}\right]^{l},
$$

for $k=1$ and $l=3$, we have in calculating (2) to convert them into exponential series,

$$
T=Z^{-N / 2},
$$

with $N=1$. We meet further the value $N=2$. This problem can be solved by wellknown methods. The initial value of $T$ corresponds to the unperturbed circular orbits $\left(p_{i}=0\right)$, that is, for (10) $T_{0}=1$ and for $(11)$,

$$
T_{0}=\gamma^{(i j)}\left\{\begin{array}{lllll}
0, & -\frac{1}{2} k N, & -\frac{1}{2} l N, & 0, & a_{j} / a_{i} \\
0, & -\frac{1}{2} l N, & -\frac{1}{2} k N, & \frac{1}{2}(k-l) N, & a_{i} / a_{j}
\end{array}\right\}
$$

with function $\gamma^{(i j)}(n, x, y, v, \alpha)$ determined in Brumberg (1970). 


\section{Equations of Motion}

Putting $p_{i}=p_{i}^{(0)}+\delta p_{i}$ and collecting in (2) and (3) the linear terms we obtain

$$
\begin{aligned}
& P_{i}=P_{i}^{(0)}-\sum_{j=1}^{N}\left(K_{i j} \delta p_{j}+L_{i j} \delta q_{j}\right)+P_{i}^{*}, \\
& W_{i}=-\sum_{j=1}^{N} M_{i j} w_{j}+W_{i}^{*} .
\end{aligned}
$$

The unknowns $\delta p_{i}$ and $w_{i}$ must obey the equations

$$
\begin{aligned}
& \delta \ddot{p}_{i}+2 \sqrt{ }-1 n_{i} \delta \dot{p}_{i}+n_{i}^{2} \sum_{j=1}^{N}\left[\left(-\frac{3}{2} \delta_{i j}+K_{i j}\right) \delta p_{j}+\left(-\frac{3}{2} \delta_{i j}+L_{i j}\right) \delta q_{j}\right]=n_{i}^{2} P_{i}^{*}, \\
& \ddot{w}_{i}+n_{i}^{2} \sum_{j=1}^{N}\left(\delta_{i j}+M_{i j}\right) w_{j}=n_{i}^{2} W_{i}^{*} .
\end{aligned}
$$

Assuming (6) to be known one can find functions depending on intermediary as follows:

$$
\begin{aligned}
& A_{i}=\left(1-p_{i}\right)\left(\frac{a_{i}}{r_{i}}\right)^{5}, \quad B_{i}=\left(\frac{a_{i}}{r_{i}}\right)^{3}, \quad C_{i}=\left(1-p_{i}\right)\left(\frac{a_{i}}{r_{i}}\right)^{3}, \\
& D_{i}=\left(1-q_{i}\right)\left(\frac{a_{i}}{r_{i}}\right)^{2}, \quad E_{i}=\left(\frac{a_{i}}{r_{i}}\right)^{2}, \quad F_{i}=\left(1-p_{i}\right)^{2}\left(\frac{a_{i}}{r_{i}}\right)^{5}, \\
& A_{i j}=\left[\frac{a_{i}}{a_{i j}}\left(1-p_{i}\right)-\frac{a_{j}}{a_{i j}}\left(1-p_{j}\right) \zeta_{i j}^{-1}\right]\left(\frac{a_{i j}}{\Delta_{i j}}\right)^{5}, \quad B_{i j}=\left(\frac{a_{i j}}{\Delta_{i j}}\right)^{3}, \\
& C_{i j}=\left[\frac{a_{i}}{a_{i j}}\left(1-p_{i}\right)-\frac{a_{j}}{a_{i j}}\left(1-p_{j}\right) \zeta_{i j}^{-1}\right]\left(\frac{a_{i j}}{\Delta_{i j}}\right)^{3}, \\
& D_{i j}=\left[\frac{a_{i}}{a_{i j}}\left(1-q_{i}\right)-\frac{a_{j}}{a_{i j}}\left(1-q_{j}\right) \zeta_{i j}\right]\left(\frac{a_{i j}}{\Delta_{i j}}\right)^{2}, \quad E_{i j}=\left(\frac{a_{i j}}{\Delta_{i j}}\right)^{2}, \\
& F_{i j}=\left[\frac{a_{i}}{a_{i j}}\left(1-p_{i}\right)-\frac{a_{j}}{a_{i j}}\left(1-p_{j}\right) \zeta_{i j}^{-1}\right]^{2}\left(\frac{a_{i j}}{\Delta_{i j}}\right)^{5} .
\end{aligned}
$$

Determination of these functions in form (6)-(8) calls for conversion (12) with (10) and (11). The corresponding values of integers $k, l$ and $N$ are

\begin{tabular}{r|cccccc} 
& $A$ & $B$ & $C$ & $D$ & $E$ & $F$ \\
\hline$k$ & 3 & 3 & 1 & 1 & 1 & 1 \\
$l$ & 5 & 3 & 3 & 0 & 1 & 5 \\
$N$ & 1 & 1 & 1 & 2 & 2 & 1.
\end{tabular}

One may use the check relations

$$
A_{i}=C_{i} E_{i}, \quad B_{i}=C_{i} D_{i}, \quad A_{i}=D_{i} F_{i},
$$


and the similar expressions for two-indices functions.

With the aid of these functions the coefficients for the left members of $(15)$ can be obtained in form (6)-(8) as follows:

$$
\begin{aligned}
& K_{i i}=\frac{1}{2}\left[1-B_{i}-\mu \sum_{j=1}^{N} \kappa_{i j}\left(\frac{a_{i}}{a_{i j}}\right)^{3} B_{i j}\right], \\
& K_{i j}=\frac{1}{2} \mu \kappa_{i j}\left[\left(\frac{a_{i}}{a_{i j}}\right)^{2} \frac{a_{j}}{a_{i j}} B_{i j}-\left(\frac{a_{i}}{a_{j}}\right)^{2} B_{j}\right] \zeta_{i j}^{-1}, \quad(i \neq j) \\
& L_{i i}=\frac{3}{2}\left[1-F_{i}-\mu \sum_{j=1}^{N} \kappa_{i j}\left(\frac{a_{i}}{a_{i j}}\right)^{3} F_{i j}\right], \\
& L_{i j}=\frac{3}{2} \mu \kappa_{i j}\left[\left(\frac{a_{i}}{a_{i j}}\right)^{2} \frac{a_{j}}{a_{i j}} \zeta_{i j} F_{i j}-\left(\frac{a_{i}}{a_{j}}\right)^{2} \zeta_{i j}^{-1} F_{j}\right], \quad(i \neq j) \\
& M_{i i}=-2 K_{i i}, \quad M_{i j}=-2 K_{i j} \zeta_{i j} \quad(i \neq j) .
\end{aligned}
$$

Put, further,

$$
\begin{aligned}
S_{i}= & D_{i} \delta p_{i}+\bar{D}_{i} \delta q_{i}-E_{i}\left(\delta p_{i} \delta q_{i}+w_{i}^{2}\right) \\
S_{i j}= & D_{i j}\left(\frac{a_{i}}{a_{i j}} \delta p_{i}-\frac{a_{j}}{a_{i j}} \zeta_{i j}^{-1} \delta p_{j}\right)+\bar{D}_{i j}\left(\frac{a_{i}}{a_{i j}} \delta q_{i}-\frac{a_{j}}{a_{i j}} \zeta_{i j} \delta q_{j}\right)- \\
& -E_{i j}\left[\left(\frac{a_{i}}{a_{i j}} \delta p_{i}-\frac{a_{j}}{a_{i j}} \zeta_{i j}^{-1} \delta p_{j}\right)\left(\frac{a_{i}}{a_{i j}} \delta q_{i}-\frac{a_{j}}{a_{i j}} \zeta_{i j} \delta q_{j}\right)+\left(\frac{a_{i}}{a_{i j}} w_{i}-\frac{a_{j}}{a_{i j}} w_{j}\right)^{2}\right]
\end{aligned}
$$

and let $T_{i}$ and $T_{i j}$ be the series of the same kind

$$
T_{i}=\sum_{k=2}^{\infty} \frac{(3 / 2)_{k}}{(1)_{k}} S_{i}^{k}, \quad T_{i j}=\sum_{k=2}^{\infty} \frac{(3 / 2)_{k}}{(1)_{k}} S_{i j}^{k}
$$

In virtue of relations

$$
\begin{array}{ll}
\left(\frac{r_{i}}{a_{i}}\right)^{2}=\frac{1-S_{i}}{E_{i}}, & \left(\frac{a_{i}}{r_{i}}\right)^{3}=B_{i}\left(1+\frac{3}{2} S_{i}+T_{i}\right), \\
\left(\frac{\Delta_{i j}}{a_{i j}}\right)^{2}=\frac{1-S_{i j}}{E_{i j}}, & \left(\frac{a_{i j}}{\Delta_{i j}}\right)^{3}=B_{i j}\left(1+\frac{3}{2} S_{i j}+T_{i j}\right),
\end{array}
$$

there results

$$
\begin{aligned}
P_{i}^{*}= & -\frac{3}{2} A_{i}\left(\delta p_{i} \delta q_{i}+w_{i}^{2}\right)-\frac{3}{2} B_{i} S_{i} \delta p_{i}+\left(C_{i}-B_{i} \delta p_{i}\right) T_{i}+ \\
& +\mu \sum_{j=1}^{N} \cdot\left(\kappa _ { i j } \left\{-\frac{3}{2} A_{i j}\left[\left(\frac{a_{i}}{a_{i j}} \delta p_{i}-\frac{a_{j}}{a_{i j}} \zeta_{i j}^{-1} \delta p_{j}\right)\left(\frac{a_{i}}{a_{i j}} \delta q_{i}-\frac{a_{j}}{a_{i j}} \zeta_{i j} \delta q_{j}\right)+\right.\right.\right. \\
& \left.+\left(\frac{a_{i}}{a_{i j}} w_{i}-\frac{a_{j}}{a_{i j}} w_{j}\right)^{2}\right]-\frac{3}{2} B_{i j} S_{i j}\left(\frac{a_{i}}{a_{i j}} \delta p_{i}-\frac{a_{j}}{a_{i j}} \zeta_{i j}^{-1} \delta p_{j}\right)+
\end{aligned}
$$




$$
\begin{aligned}
+ & {\left[C_{i j}-B_{i j}\left(\frac{a_{i}}{a_{i j}} \delta p_{i}-\frac{a_{j}}{a_{i j}} \zeta_{i j}^{-1} \delta p_{j}\right)\right] T_{i j}+\zeta_{i j}^{-1}\left(\frac{a_{i}}{a_{j}}\right)^{2} \times } \\
& \left.\times\left[-\frac{3}{2} A_{j}\left(\delta p_{j} \delta q_{j}+w_{j}^{2}\right)-\frac{3}{2} B_{j} S_{j} \delta p_{j}+\left(C_{j}-B_{j} \delta p_{j}\right) T_{j}\right]\right\}, \\
W_{i}^{*}= & -B_{i}\left(\frac{3}{2} S_{i}+T_{i}\right) w_{i}-\mu \sum_{j=1}^{N} \kappa_{i j}\left[B_{i j}\left(\frac{3}{2} S_{i j}+T_{i j}\right)\left(\frac{a_{i}}{a_{i j}} w_{i}-\frac{a_{j}}{a_{i j}} w_{j}\right)+\right. \\
& \left.+\left(\frac{a_{i}}{a_{j}}\right)^{2} B_{j}\left(\frac{3}{2} S_{j}+T_{j}\right) w_{j}\right] .
\end{aligned}
$$

These expressions allow us to calculate the right members without expansions in powers of $\delta p_{i}, \delta q_{i}$ and $w_{i}$.

\section{Linear Inequalities}

According to the general theory the solution of (15) can be presented in the form

$$
\begin{aligned}
& \delta p_{i}=\sum p_{p q r s}^{(i)} \prod_{j=1}^{N} a_{j}^{p_{j}} \bar{a}_{j}^{q_{j}} b_{j}^{r_{j}} \bar{b}_{j}^{s_{j}}, \\
& w_{i}=\sum w_{p q r s}^{(i)} \prod_{j=1}^{N} a_{j}^{p_{j}} \bar{a}_{j}^{q_{j}} b_{j}^{r_{j}} \bar{b}_{j}^{s_{j}},
\end{aligned}
$$

where summation goes over all nonnegative values of indices $p_{j}, q_{j}, r_{j}$ and $s_{j}$, with positive sum. The sum of all $r_{j}$ and $s_{j}$ is even in (22) and odd in (23). Coefficients $p_{p q r s}^{(i)}$ and $w_{p q r s}^{(i)}$ are series of form (6)-(8). The variables $a_{j}$ and $b_{j}$ (the same designation $a_{j}$ for the unknown variables and semimajor axes should not lead to misunderstanding) are related with slow variables $\alpha_{j}, \beta_{j}$ by simple expressions,

$$
a_{j}=\alpha_{j} \exp \sqrt{ }-1 \lambda_{j}, \quad b_{j}=\beta_{j} \exp \sqrt{ }-1 \lambda_{j} .
$$

The unknowns $\alpha_{j}$ and $\beta_{j}$ represent a sort of eccentricity and obliquity variables, respectively. For the two-body problem they can be expressed in terms of the usual Keplerian elements (Brumberg and Chapront, 1974). The secular system determining these variables is treated in Section 6.

Determination of the linear terms of $(22)$ and (23) is performed in the following way. Let $Q=\left\|Q_{\kappa v}\right\|(\kappa, v=1,2, \ldots, 6)$ be a block matrix, each block representing linear combination of matrices $K=\left\|K_{i j}\right\|, L=\left\|L_{i j}\right\|$ and $M=\left\|M_{i j}\right\|$,

$$
\begin{aligned}
& Q_{11}=K-L-\bar{K}+\bar{L}, \quad Q_{12}=-\frac{2}{3}(K+L+\bar{K}+\bar{L}), \\
& Q_{13}=\frac{1}{2}(-K+3 L+3 \bar{K}-\bar{L}), \quad Q_{21}=\frac{3}{2}(-K+L-\bar{K}+\bar{L}), \\
& Q_{22}=K+L-\bar{K}-\bar{L}, \quad Q_{23}=\frac{3}{4}(K-3 L+3 \bar{K}-\bar{L}), \\
& Q_{3 v}=\frac{1}{2} Q_{1 v}+\frac{2}{3} Q_{2 v} \quad(v=1,2,3), \quad Q_{43}=-\frac{1}{2} Q_{13}+\frac{2}{3} Q_{23}, \\
& Q_{55}=-Q_{65}=\frac{1}{2} M .
\end{aligned}
$$


Eight blocks of $Q$ are defined by conjugate relations,

$$
\begin{array}{lr}
Q_{14}=\bar{Q}_{13}, & Q_{24}=-\bar{Q}_{23}, \quad Q_{41}=\bar{Q}_{31}, \quad Q_{42}=-\bar{Q}_{32}, \\
Q_{34}=-\bar{Q}_{43}, & Q_{44}=-\bar{Q}_{33}, \quad Q_{56}=-\bar{Q}_{65}, \quad Q_{66}=-\bar{Q}_{55} .
\end{array}
$$

Remaining 16 blocks are zero matrices (for values $\kappa=1,2,3,4, v=5,6$ and $\kappa=5,6$, $v=1,2,3,4)$.

Hence, each element $Q_{\kappa v}[i, j](\kappa, v=1,2, \ldots, 6 ; i, j=1,2, \ldots, N)$ of $Q$ can be found in form (6)-(8), that is,

$$
Q_{\kappa v}[i, j]=\sum Q_{\gamma}^{(\kappa v i j)} \exp \sqrt{ }-1(\gamma \lambda)
$$

Matrices $G$ and $S$ needed for calculation of the linear inequalities are of the same form as $Q$. Relations (26) remain valid for the blocks of $G$. The blocks of $S$ satisfy similar relations but with the change of signs,

$$
\begin{aligned}
& S_{14}=-\bar{S}_{13}, \quad S_{24}=\bar{S}_{23}, \quad S_{41}=-\bar{S}_{31}, \quad S_{42}=\bar{S}_{32}, \\
& S_{34}=\bar{S}_{43}, \quad S_{44}=\bar{S}_{33}, \quad S_{56}=\bar{S}_{65}, \quad S_{66}=\bar{S}_{55} .
\end{aligned}
$$

Therefore, each of these matrices is completely defined by 12 blocks enumerated in (25), and it is sufficient to give expressions only for these blocks. $G$ and $S$ satisfy equations

$$
\begin{aligned}
& G=Q(E+S)-\mathcal{N}^{-1} S \mathcal{N} G^{*} \\
& G=G^{*}+\tilde{G} \\
& \dot{S}+\sqrt{ }-1(S \mathscr{N} P-\mathscr{N} P S)=\sqrt{ }-1 \mathscr{N} \tilde{G}
\end{aligned}
$$

where $\mathcal{N}=\operatorname{diag}\left(n_{1}, n_{2}, \ldots, n_{N}\right)$ and $P=\left\|P_{\kappa v}\right\|$ being a constant block matrix with nonzero blocks,

$$
P_{12}=P_{33}=P_{55}=E, \quad P_{44}=P_{66}=-E .
$$

$E$ denotes the unit matrix. $G$ and $S$, like $Q$, have the factor of planetary masses and can be found from (29) $-(31)$ by iterations. Let us assume that we know the approximate values of elements $G$ and $S$ in the form of (27). Then (29) yields more accurate series for $G$ :

$$
\begin{aligned}
G_{\kappa \nu}[i, j]= & Q_{\kappa \nu}[i, j]+\sum_{k=1}^{N} \sum_{\lambda=1}^{6}\left(Q_{\kappa \lambda}[i, k] S_{\lambda \nu}[k, j)\right]- \\
& \left.-\frac{n_{k}}{n_{i}} S_{\kappa \lambda}[i, k] G_{\lambda \nu}^{*}[k, j]\right) .
\end{aligned}
$$

We collect in $G^{*}$ the terms with coefficients $G_{\gamma}^{(\kappa v i j)}$ such that

$$
\begin{aligned}
& \gamma_{k}=0, \quad \text { for } \quad(\kappa, v)=(1,1),(1,2),(2,1),(2,2), \\
& \gamma_{k}=\delta_{k i}-\delta_{k j}, \quad \text { for } \quad(\kappa, v)=(3,3),(5,5) \quad(k=1,2, \ldots, N) .
\end{aligned}
$$


Remaining terms are included into $\widetilde{G}$. From (31) we obtain the improved series for $S$ :

$$
\begin{array}{ll}
S_{\gamma}^{(21 i j)}=\frac{n_{i}}{(\gamma n)} G_{\gamma}^{(21 i j)}, \quad S_{\gamma}^{(22 i j)}=\frac{n_{i}}{(\gamma n)}\left(G_{\gamma}^{(22 i j)}-\frac{n_{j}}{n_{i}} S_{\gamma}^{(21 i j)}\right), \\
S_{\gamma}^{(11 i j)}=\frac{n_{i}}{(\gamma n)}\left(G_{\gamma}^{(11 i j)}+S_{\gamma}^{(21 i j)}\right), \quad S_{\gamma}^{(12 i j)}=\frac{n_{i}}{(\gamma n)}\left(G_{\gamma}^{(12 i j)}-\frac{n_{j}}{n_{i}} S_{\gamma}^{(11 i j)}+S_{\gamma}^{(22 i j)}\right), \\
S_{\gamma}^{(23 i j)}=\frac{n_{i}}{(\gamma n)+n_{j}} G_{\gamma}^{(23 i j)}, \quad S_{\gamma}^{(13 i j)}=\frac{n_{i}}{(\gamma n)+n_{j}}\left(G_{\gamma}^{(13 i j)}+S_{\gamma}^{(23 i j)}\right), \\
S_{\gamma}^{(31 i j)}=\frac{n_{i}}{(\gamma n)-n_{i}} G_{\gamma}^{(31 i j)}, \quad S_{\gamma}^{(32 i j)}=\frac{n_{i}}{(\gamma n)-n_{i}}\left(G_{\gamma}^{(32 i j)}-\frac{n_{j}}{n_{i}} S_{\gamma}^{(31 i j)}\right), \\
S_{\gamma}^{(\kappa v i j)}=\frac{n_{i}}{(\gamma n)+n_{j}-n_{i}} G_{\gamma}^{(\kappa v i j)} \quad(\kappa, v)=(3,3),(5,5), \\
S_{\gamma}^{(\kappa v i j)}=\frac{n_{i}}{(\gamma n)+n_{j}+n_{i}} G_{\gamma}^{(\kappa v i j)} \quad(\kappa, v)=(4,3),(6,5) .
\end{array}
$$

The zero values of divisors correspond to critical terms (33) which are absent in $\tilde{G}$. The associated terms of $S$ are taken as zero. The initial values of this iteration process are

$$
G=Q, \quad S=0
$$

Having found $G$ and $S$ we compute the linear terms in the coordinates with the aid of

$$
\begin{aligned}
& \delta p_{i}^{(1)}=\sum_{j=1}^{N}\left[\left(-\frac{1}{2} \delta_{i j}+c_{i j}\right) a_{j}+\left(\frac{3}{2} \delta_{i j}+d_{i j}\right) \bar{a}_{j}\right], \\
& w_{i}^{(1)}=\sum_{j=1}^{N}\left[\left(\delta_{i j}+f_{i j}\right) b_{j}+\left(\delta_{i j}+\bar{f}_{i j}\right) \bar{b}_{j}\right],
\end{aligned}
$$

where $c_{i j}, d_{i j}$ and $f_{i j}$ represent elements of matrices,

$$
\begin{aligned}
& c=S_{13}-\frac{2}{3} S_{23}-\frac{1}{2} S_{33}+\frac{3}{2} S_{43}, \\
& d=-S_{13}-\frac{2}{3} S_{23}+\frac{3}{2} \bar{S}_{33}-\frac{1}{2} S_{43}, \\
& f=S_{55}+S_{65},
\end{aligned}
$$

and hence are available in form (6)-(8). The components of velocities can be obtained, if necessary, from

$$
\begin{aligned}
\delta \dot{p}_{i}^{(1)} & =\sqrt{ }-1 n_{i} \sum_{j=1}^{N}\left[\left(-\frac{1}{2} \delta_{i j}+c_{i j}^{\prime}\right) a_{j}+\left(-\frac{3}{2} \delta_{i j}+d_{i j}^{\prime}\right) \bar{a}_{j}\right], \\
\dot{w}_{i}^{(1)} & =\sqrt{ }-1 n_{i} \sum_{j=1}^{N}\left[\left(\delta_{i j}+f_{i j}^{\prime}\right) b_{j}-\left(\delta_{i j}+\bar{f}_{i j}^{\prime}\right) b_{j}\right],
\end{aligned}
$$


where

$$
\begin{aligned}
& c^{\prime}=S_{23}-\frac{1}{2} S_{33}-\frac{3}{2} S_{43}, \\
& d^{\prime}=\bar{S}_{23}-\frac{3}{2} \bar{S}_{33}-\frac{1}{2} \bar{S}_{43}, \\
& f^{\prime}=S_{55}-S_{65} .
\end{aligned}
$$

This completes the computation of inequalities linear in eccentricities and inclinations. Just as in the case of intermediary we have to deal here only with operations on exponential series of form (6)-(8).

\section{Nonlinear Inequalities}

In accordance with the general theory the nonlinear problem reduces to the determination of vectors $U$ and $\Gamma$ from the equations,

$$
\begin{aligned}
& U=R+Q \Gamma-\mathcal{N}^{-1} \Gamma_{y} \mathcal{N} G^{*} Y-\mathscr{N}^{-1}\left(S+\Gamma_{y}\right) \mathscr{N} U^{*}, \\
& U=U^{*}+\tilde{U} \\
& \Gamma_{t}+\sqrt{-1}\left(\Gamma_{y} \mathscr{N} P Y-\mathcal{N} P \Gamma\right)=\sqrt{-1} \mathscr{N} \tilde{U}
\end{aligned}
$$

Vectors $U$ and $\Gamma$ consist of six subvectors $U_{\kappa}$ and $\Gamma_{\kappa}(\kappa=1,2, \ldots, 6)$ respectively. Herein

$$
\begin{array}{lc}
\bar{U}_{1}=U_{1}, & \bar{U}_{2}=-U_{2}, \quad U_{4}=-\bar{U}_{3}, \quad U_{6}=-\bar{U}_{5}, \\
\bar{\Gamma}_{1}=-\Gamma_{1}, & \bar{\Gamma}_{2}=\Gamma_{2}, \quad \Gamma_{4}=\bar{\Gamma}_{3}, \quad \Gamma_{6}=\bar{\Gamma}_{5} .
\end{array}
$$

Each subvector has $N$ components. Vectors of variables $Y$ and right members $R$ have the same properties as $\Gamma$ and $U$, respectively. Their components are

$$
\begin{array}{ll}
Y_{1}[i]=Y_{2}[i]=0, & Y_{3}[i]=a_{i}, \quad Y_{5}[i]=b_{i}, \\
R_{1}[i]=-P_{i}^{*}-\bar{P}_{i}^{*}, & R_{2}[i]=\frac{3}{2}\left(P_{i}^{*}-\bar{P}_{i}^{*}\right), \\
R_{3}[i]=\frac{1}{2} P_{i}^{*}-\frac{3}{2} \bar{P}_{i}^{*}, & R_{5}[i]=-\frac{1}{2} W_{i}^{*} .
\end{array}
$$

Rewriting (39) in scalar form we obtain

$$
\begin{aligned}
U_{\kappa}[i]= & R_{\kappa}[i]+\sum_{j=1}^{N} \sum_{v=1}^{6} Q_{\kappa v}[i, j] \Gamma_{v}[j]-\sum_{j=1}^{N} \sum_{v=3}^{6} \frac{n_{j}}{n_{i}} \times \\
& \times\left\{\left(S_{\kappa v}[i, j]+\frac{\partial \Gamma_{\kappa}[i]}{\partial Y_{v}[j]}\right) U_{v}^{*}[j]+\frac{\partial \Gamma_{\kappa}[i]}{\partial Y_{v}[j]} \sum_{k=1}^{N} G_{v v}^{*}[j, k] Y_{v}[k]\right\}
\end{aligned}
$$

In virtue of (42) and (43) for determination of $U$ and $\Gamma$ only subvectors with numbers $\kappa=1,2,3,5$ are needed.

The terms of the first order in eccentricities and inclinations found in the previous section serve as initial values in determining the second-order terms, etc. In general, 
let expansions (22) and (23) be known up to order $m-1$ inclusively $(m=2,3, \ldots)$. Then the terms of order $m$ can be found in the following way:

Expressions (17) and (18) give $S_{i}$ and $S_{i j}$ up to order $m-1$. Restricting summation in (19) to value $k=m$ we find $T_{i}$ and $T_{i j}$ up to terms of order $m$. With obtained expressions of $S_{i}, S_{i j}, T_{i}$ and $T_{i j}$ the terms of order $m$ in the right members of the equations of motion result from (20) and (21). Let superscript $m$ denote the terms of order $m$ in the appropriate function. With $P_{i}^{*(m)}$ and $W_{i}^{*(m)}$ we immediately obtain from (44) the components $R_{\kappa}^{(m)}[i]$. Then the corresponding terms of $U$ and $\Gamma$ can be found by iterations. Let us assume that we have the approximate values for coefficients $U_{p q r s \gamma}^{(\kappa i)}$ and $\Gamma_{p q r s \gamma}^{(\kappa i)}$ of series

$$
U_{\kappa}^{(m)}[i]=\sum U_{p q r s}^{(\kappa i)} \prod_{j=1}^{N} a_{j}^{p_{j}} \bar{a}_{j}^{q_{j}} b_{j}^{r_{j}} \bar{b}_{j}^{s_{j}}, \quad U_{p q r s}^{(\kappa i)}=\sum U_{p q r s \gamma}^{(\kappa i)} \exp \sqrt{ }-1(\gamma \lambda),
$$

and similar ones for $\Gamma_{\kappa}^{(m)}[i]$. Indices $\gamma$ are again related here by (8). The sum of all indices $p_{j}, q_{j}, r_{j}$ and $s_{j}$ is equal to $m$. With respect to all $b_{j}$ and $b_{j}$ the series for $\kappa=1,2,3$ are even, that for $\kappa=5$ is odd. The improved values for coefficients $U_{p q r s \gamma}^{(\kappa i)}$ result from (45) or in more detail:

$$
\begin{aligned}
U_{\kappa}^{(m)}[i]= & R_{\kappa}^{(m)}[i]+\sum_{j=1}^{N} \sum_{v=1}^{6} Q_{\kappa v}[i, j] \Gamma_{v}^{(m)}[j]-\sum_{j=1}^{N} \frac{n_{j}}{n_{i}} \times \\
& \times\left\{\sum_{v=3}^{6} S_{\kappa v}[i, j] U_{v}^{*(m)}[j]+\sum_{k=1}^{N}\left(G_{33}^{*}[j, k] a_{k} \frac{\partial}{\partial a_{j}}-\right.\right. \\
& \left.-\bar{G}_{33}^{*}[j, k] \bar{a}_{k} \frac{\partial}{\partial \bar{a}_{j}}+G_{55}^{*}[j, k] b_{k} \frac{\partial}{\partial b_{j}}-\bar{G}_{55}^{*}[j, k] b_{k} \frac{\partial}{\partial \bar{b}_{j}}\right) \times \\
& \times \Gamma_{\kappa}^{(m)}[i]+\sum_{l=1}^{E[(m-2) / 2]}\left(U_{3}^{*(2 l+1)}[j] \frac{\partial}{\partial a_{j}}-\bar{U}_{3}^{*(2 l+1)}[j] \frac{\partial}{\partial \bar{a}_{j}}+\right. \\
& \left.\left.+U_{5}^{*(2 l+1)}[j] \frac{\partial}{\partial b_{j}}-\bar{U}_{5}^{*(2 l+1)}[j] \frac{\partial}{\partial \bar{b}_{j}}\right) \Gamma_{\kappa}^{(m-2 l)}[i]\right\} .
\end{aligned}
$$

Functions $U_{\kappa}^{*}[i](\kappa=3,5)$ represent the critical terms of $U_{\kappa}[i]$, that is, the terms with odd values of $m(m=3,5, \ldots)$, and the following relations for indices hold:

$$
\sum_{j=1}^{N}\left(p_{j}-q_{j}+r_{j}-s_{j}\right)=1, \quad \gamma_{j}=\delta_{i j}-p_{j}+q_{j}-r_{j}+s_{j}
$$

For even values of $m(m=2,4, \ldots)$ the critical terms satisfying relations

$$
\sum_{j=1}^{N}\left(p_{j}-q_{j}+r_{j}-s_{j}\right)=0, \quad \gamma_{j}=-p_{j}+q_{j}-r_{j}+s_{j}
$$

might have generally occurred in subvectors $U_{1}$ and $U_{2}$. But $U_{1}^{*}=0$ if the critical terms in $\Gamma_{2}$ are chosen appropriately. Relation $U_{2}^{*}=0$ holds true by itself and serves as a reliable check of calculations. Then the improved values for coefficients $\Gamma_{p q r s \gamma}^{(\kappa i)}$ 
are determined by

$$
\begin{aligned}
& \Gamma_{p q r s \gamma}^{(2 i)}=\frac{n_{i}}{((p-q+r-s+\gamma) n)} U_{p q r s \gamma}^{(2 i)}, \\
& \Gamma_{p q r s \gamma}^{(1 i)}=\frac{n_{i}}{((p-q+r-s+\gamma) n)}\left(U_{p q r s \gamma}^{(1 i)}+\Gamma_{p q r s \gamma}^{(2 i)}\right) ; \\
& \Gamma_{p q r s \gamma}^{(\kappa i)}=\frac{n_{i}}{((p-q+r-s+\gamma) n)-n_{i}} U_{p q r s \gamma}^{(\kappa i)} \quad(\kappa=3,5) .
\end{aligned}
$$

For critical values (47) and (48), which lead to zero divisors, the coefficients are chosen as follows:

$$
\Gamma_{p q r s \gamma}^{(2 i)}=-U_{p q r s \gamma}^{(1 i)}, \quad \Gamma_{p q r s \gamma}^{(\kappa i)}=0 \quad(\kappa=1,3,5) .
$$

The process of determining $U$ and $\Gamma$ is repeated until the desired accuracy is achieved. The initial conditions in the first application of $(46)$ are zero values for $\Gamma^{(m)}$ and $U^{*(m)}$.

The terms of order $m$ in $\delta p_{i}$ and $w_{i}$ and, if necessary, in $\delta \dot{p}_{i}$ and $\dot{w}_{i}$ can be found from expressions

$$
\begin{aligned}
\delta p_{i}^{(m)} & =\Gamma_{1}^{(m)}[i]-\frac{2}{3} \Gamma_{2}^{(m)}[i]-\frac{1}{2} \Gamma_{3}^{(m)}[i]+\frac{3}{2} \bar{\Gamma}_{3}^{(m)}[i], \\
w_{i}^{(m)} & =\Gamma_{5}^{(m)}[i]+\bar{\Gamma}_{5}^{(m)}[i], \\
\delta \dot{p}_{i}^{(m)} & =\sqrt{ }-1 n_{i}\left(\Gamma_{2}^{(m)}[i]-\frac{1}{2} \Gamma_{3}^{(m)}[i]-\frac{3}{2} \bar{\Gamma}_{3}^{(m)}[i]\right), \\
\dot{w}_{i}^{(m)} & =\sqrt{ }-1 n_{i}\left(\Gamma_{5}^{(m)}[i]-\bar{\Gamma}_{5}^{(m)}[i]\right) .
\end{aligned}
$$

Thus, expansions (22) and (23) become available up to order $m$ and one can proceed to the next order.

Calculation of the nonlinear terms described here calls for performing literal operations on computer. At present, several systems of operations on Poisson series are known, for instance Rom (1970, 1971), Broucke and Carthwaite (1969), Jefferys (1970, 1972), Kovalevsky (1971), Cherniack (1973). Such a system may be applied without much effort to the general planetary theory. Basic series (22) and (23) are arranged in increasing order of power indices. The coefficients of these power series are exponential series of form (6)-(8). They are arranged in increasing order of exponential indices or in decreasing order of magnitude of numerical coefficients. Having approximate estimates for absolute values of $a_{j}$ and $b_{j}$ it is useful to evaluate the absolute value of the whole power term. This permits us to judge the accuracy needed in calculating the corresponding exponential series. It is evident that not all possible combinations of power indices occur actually in (22) and (23). As in any planetary theory the main difficulties arise here in calculating the resonant terms due to the close commensurabilities of mean motions. Existence of such terms allow us to calculate the right members with an accuracy far greater than the accuracy of the final series for the coordinates. 


\section{Secular System}

Equations for slow variables $\alpha_{j}$ and $\beta_{j}$ have the form

$$
\begin{aligned}
& \dot{\alpha}=\sqrt{ }-1 \mathscr{N}[A \alpha+\Phi(\alpha, \bar{\alpha}, \beta, \bar{\beta})], \\
& \dot{\beta}=\sqrt{ }-1 \mathscr{N}[B \beta+\Psi(\alpha, \bar{\alpha}, \beta, \bar{\beta})] .
\end{aligned}
$$

Matrices $A$ and $B$ and vectors $\Phi$ and $\Psi$ are derived from $G_{33}^{*}, G_{55}^{*}, U_{3}^{*}$ and $U_{5}^{*}$, respectively. There results

and

$$
\begin{aligned}
& A[i, i]=G_{33}^{*}[i, i], \quad B[i, i]=G_{55}^{*}[i, i], \\
& A[i, j]=G_{33}^{*}[i, j] \zeta_{i j}^{-1}, \quad B[i, j]=G_{55}^{*}[i, j] \zeta_{i j}^{-1}, \quad(i \neq j),
\end{aligned}
$$

$$
\begin{aligned}
& \Phi_{i}=\sum^{*} U_{p q r s \gamma}^{(3 i)} \prod_{j=1}^{N} \alpha_{j}^{p_{j}} \bar{\alpha}_{j}^{q_{j}} \beta_{j}^{r_{j}} \bar{\beta}_{j}^{s_{j}}, \\
& \Psi_{i}=\sum^{*} U_{p q r s \gamma}^{(5 i)} \prod_{j=1}^{N} \alpha_{j}^{p_{j}} \bar{\alpha}_{j}^{q_{j}} \beta_{j}^{r_{j}} \bar{\beta}_{j}^{s_{j}} .
\end{aligned}
$$

In (55) an asterisk denotes summation over critical values (47).

Solution of the autonomous system (53) can be performed by further application of Birkhoff's normalization. Iterations with respect to planetary masses are not needed anymore. In this solution $\alpha_{i}$ and $\beta_{i}$ are presented by series of odd power forms relative to $u_{j}, \bar{u}_{j}, v_{j}$ and $\bar{v}_{j}$, where

$$
u_{j}=\xi_{j} \exp \sqrt{ }-1\left(c_{j} t+\tau_{j}\right), \quad v_{j}=\eta_{j} \exp \sqrt{ }-1\left(g_{j} t+\chi_{j}\right),
$$

$\xi_{j}, \eta_{j}, \tau_{j}$ and $\chi_{j}$ being real constants. Frequencies $c_{j}$ and $g_{j}$ are series in powers of $\xi_{k}^{2}$ and $\eta_{k}^{2}$. Substitution of these expressions for $\alpha_{i}$ and $\beta_{i}$ into (24), (22) and (23) would lead to the planetary theory in trigonometric form. But such a form is not suitable for the practical aims. It is more preferable to compute $\alpha_{i}$ and $\beta_{i}$ for some interval of time and to substitute these constant numerical values into (22) and (23). Then the coordinates and velocities of the planets will be expressed by exponential series relative to the mean longitudes. For the next interval of time new numerical values of $\alpha_{i}$ and $\beta_{i}$ must be used. In such numerical computation, instead of Birkhoff's normalization, it is possible to apply a polynomial representation of $\alpha_{i}$ and $\beta_{i}$ in powers of time. For this purpose we may substitute into (53) the power series

$$
\alpha_{i}=\sum_{k=0}^{\infty} \alpha_{k}^{(i)} t^{k}, \quad \beta_{i}=\sum_{k=0}^{\infty} \beta_{k}^{(i)} t^{k}
$$

with complex coefficients. Coefficients $\alpha_{0}^{(i)}$ and $\beta_{0}^{(i)}$ are arbitrary constants. The recurrence relations,

$$
\begin{aligned}
& (k+1) \alpha_{k+1}^{(i)}=\sqrt{ }-1 n_{i}\left(\sum_{j=1}^{N} A_{i j} \alpha_{k}^{(j)}+\Phi_{k}^{(i)}\right), \\
& (k+1) \beta_{k+1}^{(i)}=\sqrt{ }-1 n_{i}\left(\sum_{j=1}^{N} B_{i j} \beta_{k}^{(j)}+\Psi_{k}^{(i)}\right),
\end{aligned}
$$


allow us to obtain all subsequent coefficients. $\Phi_{k}^{(i)}$ and $\Psi_{k}^{(i)}$ denote here the coefficients of $t^{k}$ in substituting (57) into $\Phi_{i}$ and $\Psi_{i}$. It is to be noted that in treating a similar problem Krasinsky and Pius (1971) have determined the polynomial coefficients by interpolating the results of numerical integration.

\section{Application to the Main Problem of the Galilean Satellites of Jupiter}

The method described here may be extended into the case of commensurability of mean motions and applied to the main problem of the Galilean satellites of Jupiter. In spite of several classical theories this problem can still not be regarded as exhausted. Among recent investigations one may note Marsden (1966), Ferraz-Mello (1966), Sagnier (1972). The method of the last-mentioned paper is very close to that of the general planetary theory. Therefore, we confine ourselves to some general remarks.

By a main problem is meant here the study of the motion of the Galilean satellites $(N=4)$ in the gravitational field of Jupiter considered as a point-mass. Only perturbations due to the mutual attraction of the satellites are taken into account. All other perturbations such as those caused by the Sun or the asphericity of Jupiter are of less importance and can be neglected in the main problem. The mean motions of the satellites are supposed to satisfy one resonant relation,

$$
\left(\gamma^{*} n\right)=0
$$

where $(8)$ remains valid:

$$
\gamma_{1}^{*}=1, \quad \gamma_{2}^{*}=-3, \quad \gamma_{3}^{*}=2, \quad \gamma_{4}^{*}=0 .
$$

Approximate commensurabilities $n_{1}-2 n_{2} \approx 0, n_{2}-2 n_{3} \approx 0$ are not regarded as resonance (like the commensurabilities $2 n_{5}-5 n_{6} \approx 0, n_{7}-2 n_{8} \approx 0$ in the planetary theory).

The intermediate solution $p_{i}^{(0)}$ is presented in the old form (6)-(8). By substituting these series into the right members $P_{i}^{(0)}$ we separate the resonant terms,

$$
P_{i}^{(0) *}=\sum^{*} P_{\gamma}^{(i)} \exp \sqrt{ }-1(\gamma \lambda),
$$

where an asterisk denotes summation only over the resonant values

$$
\gamma_{j}=K \gamma_{j}^{*} \quad(K= \pm 1, \pm 2, \ldots) .
$$

Let $\tilde{P}_{i}^{(0)}$ stand for the nonresonant terms of the right members. Then

$$
P_{i}^{(0)}=P_{i}^{(0) *}+\widetilde{P}_{i}^{(0)}
$$

Coefficients $p_{\gamma}^{(i)}$ can be obtained again by (9) where $P_{\gamma}^{(i)}$ represent now coefficients of the expansions for functions $\widetilde{P}_{i}^{(0)}$. As usually, coefficients $p_{\gamma}^{(i)}$ for the resonant values (61) may be set equal to zero.

The variables $\delta p_{i}$ and $w_{i}$ are defined by Equations (15) but the right member $n_{i}^{2} P_{i}^{*}$ of Equation (15a) is to be replaced now by $n_{i}^{2}\left(P_{i}^{*}+P_{i}^{(0) *}\right)$. As a result of this change 
the basic matrix equation equivalent to system (15) takes the form

$$
\dot{X}=\sqrt{ }-1 \mathscr{N}\left[(P+Q) X+R(X, t)+R^{(0)}(t)\right]
$$

where $R^{(0)}(t)$ is determined by (44) with substitution $P_{i}^{*}=P_{i}^{(0) *}$ and $W_{i}^{*}=0$. Unlike the nonresonant case one has to include into Birkhoff's transformation a term $\Gamma^{(0)}(t)$, depending on time only. Thus we find that the transformation

$$
X=(E+S) Y+\Gamma(Y, t)+\Gamma^{(0)}(t)
$$

changes (63) into

$$
\ddot{Y}=\sqrt{ }-1 \mathscr{N}\left[H Y+F(Y, t)+F^{(0)}(t)\right],
$$

where

$$
\begin{aligned}
\sqrt{ }-1 \mathscr{N} H= & (E+S)^{-1}[\sqrt{ }-1 \mathscr{N}(P+Q)(E+S)-\dot{S}] \\
\sqrt{ }-1 \mathscr{N} F^{(0)}= & (E+S)^{-1}\left[\sqrt{ }-1 \mathscr{N} R^{(0)}+\sqrt{ }-1 \mathscr{N}(P+Q) \Gamma^{(0)}-\dot{\Gamma}^{(0)}\right] \\
\sqrt{ }-1 \mathscr{N} F= & \left(E+S+\Gamma_{y}\right)^{-1}[\sqrt{ }-1 \mathscr{N} R+\sqrt{ }-1 \mathscr{N}(P+Q) \Gamma- \\
& \left.-\sqrt{ }-1 \Gamma_{y} \mathscr{N}\left(H Y+F^{(0)}\right)-\Gamma_{t}\right]
\end{aligned}
$$

Expressions $(63)-(68)$ underlie the theory. Their only distinction from the corresponding expressions of the planetary theory (Brumberg and Chapront, 1974) consists in the presence of the terms $R^{(0)}, \Gamma^{(0)}$ and $F^{(0)}$. To bring both theories closer together impose the relation $F^{(0)}=0$. Then the presence of $R^{(0)}$ has three consequences:

(a) In constructing the linear theory the critical terms in $G_{\kappa v}[i, j]$ will be determined, instead of (33), by the following relations:

$$
\begin{aligned}
& \gamma_{k}=K \gamma_{k}^{*} \quad \text { for }(\kappa, v)=(1,1),(1,2),(2,1),(2,2), \\
& \gamma_{k}=\delta_{k i}-\delta_{k j}+K \gamma_{k}^{*} \quad \text { for } \quad(\kappa, v)=(3,3),(5,5) \\
& (k=1,2, \ldots, N ; \quad K=0, \pm 1, \pm 2, \ldots) .
\end{aligned}
$$

Zero value for $K$ corresponds to (33). The expressions for the matrices $A$ and $B$ take the form

$$
\begin{aligned}
& A[i, j]=\sum_{K=-\infty}^{\infty} G_{\gamma}^{(33 i j)} \exp \sqrt{ }-1 K\left(\gamma^{*} \lambda\right), \\
& B[i, j]=\sum_{K=-\infty}^{\infty} G_{\gamma}^{(55 i j)} \exp \sqrt{ }-1 K\left(\gamma^{*} \lambda\right),
\end{aligned}
$$

where the value of $K$ defines the resonant set of indices,

$$
\gamma_{k}=\delta_{k i}-\delta_{k j}+K \gamma_{k}^{*}
$$


(b) In the nonlinear theory the resonant parts of functions $U_{\kappa}[i]$ will include terms with indices satisfying the relations

$$
\begin{array}{r}
\sum_{j=1}^{N}\left(p_{j}-q_{j}+r_{j}-s_{j}\right)=0, \quad \gamma_{j}=-p_{j}+q_{j}-r_{j}+s_{j}+K \gamma_{j}^{*}, \\
K=0, \pm 1, \pm 2, \ldots
\end{array}
$$

for $\kappa=1,2$ and

$$
\begin{array}{r}
\sum_{j=1}^{N}\left(p_{j}-q_{j}+r_{j}-s_{j}\right)=1, \quad \gamma_{j}=\delta_{i j}-p_{j}+q_{j}-r_{j}+s_{j}+K \gamma_{j}^{*}, \\
K=0, \pm 1, \pm 2, \ldots,
\end{array}
$$

for $\kappa=3,5$. These expressions generalize (48) and (47), respectively. Therefore, nonlinear terms of the secular system will be

$$
\begin{aligned}
& \Phi_{i}=\sum_{p, q, r, s}^{*} \sum_{K=-\infty}^{\infty} U_{p q r s \gamma}^{(3 i)} \exp \sqrt{ }-1 K\left(\gamma^{*} \lambda\right) \prod_{j=1}^{N} \alpha_{j}^{p_{j}} \bar{\alpha}_{j}^{q_{j}} \beta_{j}^{r_{j}} \bar{\beta}_{j}^{s_{j}}, \\
& \Psi_{i}=\sum_{p, q, r, s}^{*} \sum_{K=-\infty}^{\infty} U_{p q r s \gamma}^{(5 i)} \exp \sqrt{ }-1 K\left(\gamma^{*} \lambda\right) \prod_{j=1}^{N} \alpha_{j}^{p_{j}} \bar{\alpha}_{j}^{q_{j}} \beta_{j}^{r_{j}} \bar{\beta}_{j}^{s_{j}},
\end{aligned}
$$

where indices $p, q, r, s$ and $\gamma$ are related by (72).

(c) Additional terms caused by the resonance and described by vector $\Gamma^{(0)}$ satisfy the equation

$$
\dot{\Gamma}^{(0)}-\sqrt{ }-1 \mathscr{N} P \Gamma^{(0)}=\sqrt{ }-1 \mathscr{N} U^{(0)},
$$

where $U^{(0)}(t)$ is determined by an iterative expression:

$$
U^{(0)}=R^{(0)}+Q \Gamma^{(0)} \text {. }
$$

There results

$$
\begin{aligned}
\dot{\Gamma}_{1}^{(0)}-\sqrt{ }-1 \mathscr{N} \Gamma_{2}^{(0)} & =\sqrt{ }-1 \mathscr{N} U_{1}^{(0)}, \\
\dot{\Gamma}_{2}^{(0)} & =\sqrt{ }-1 \mathscr{N} U_{2}^{(0)}, \\
\dot{\Gamma}_{3}^{(0)}-\sqrt{ }-1 \mathscr{N} \Gamma_{3}^{(0)} & =\sqrt{ }-1 \mathscr{N} U_{3}^{(0)} .
\end{aligned}
$$

Additional corrections $\delta p_{i}^{(0)}$ caused by $\Gamma^{(0)}(t)$ can be obtained by (51) with $m=0$. System (77) provides a complement to the secular system. If we neglect the libration of the satellites and assume the strict fulfilment of (55) and

$$
\left(\gamma^{*} \lambda\right)=180^{\circ}, \cdot
$$

then the right members $P_{i}^{(0) *}$ will be real constants,

$$
P_{i}^{(0) *}=\sum_{K=-\infty}^{\infty}(0)(-1)^{K} P_{\gamma}^{(i)}, \quad \gamma_{j}=K \gamma_{j}^{*}
$$


and up to the terms of order $\mu^{2}$ inclusively we obtain

$$
\Gamma_{1}^{(0)}[i]=0, \quad \Gamma_{2}^{(0)}[i]=2 P_{i}^{(0) *}, \quad \Gamma_{3}^{(0)}[i]=P_{i}^{(0) *}
$$

Subsequent approximations for $\Gamma_{\kappa}^{(0)}[i]$ are determined by (49) and (50) replacing $\Gamma$ and $U$ by $\Gamma^{(0)}$ and $U^{(0)}$ and putting $p=q=r=s=0$. Critical terms cannot arise in $U_{3}^{(0)}$ and in $U_{1}^{(0)}$, they vanish in virtue of the choice of the appropriate terms in $\Gamma_{2}^{(0)}$ and their absence in $U_{2}^{(0)}$ is again the check relation of the method. The secular system will be autonomous in this case and may be solved by the methods of the previous section.

Neglect of the libration which so far has not been discovered from observations signifies the choice of the particular solution with two missing arbitrary constants. Approximate consideration of the libration may be performed in the following way.

From (44) we have

$$
R_{1}^{(0)}[i]=\sum_{k=1}^{\infty} a_{k}^{(i)} \cos k\left(\gamma^{*} \lambda\right), \quad R_{2}^{(0)}[i]=\sqrt{ }-1 \sum_{k=1}^{\infty} b_{k}^{(i)} \sin k\left(\gamma^{*} \lambda\right),
$$

where

$$
a_{k}^{(i)}=-2\left(P_{\gamma}^{(i)}+P_{-\gamma}^{(i)}\right), \quad b_{k}^{(i)}=3\left(P_{\gamma}^{(i)}-P_{-\gamma}^{(i)}\right), \quad \gamma_{j}=k \gamma_{j}^{*}
$$

Supposing that (59) and (78) are close to strict fulfilment and differentiating the first Equation (77) there results:

$$
\ddot{\Gamma}_{1}^{(0)}+\mathcal{N}^{2} U_{2}^{(0)} \approx 0
$$

or up to the terms of order $\mu^{2}$ inclusively:

$$
\ddot{\Gamma}_{1}^{(0)}[i]+\sqrt{ }-1 n_{i}^{2} \sum_{k=1}^{\infty} b_{k}^{(i)} \sin k\left(\gamma^{*} \lambda\right)=0 \text {. }
$$

In the resonant case the mean longitides must have the resonant corrections $\delta \lambda_{i}$. Therefore,

$$
\lambda_{i}=\lambda_{i}^{(0)}+\delta \lambda_{i}
$$

These corrections can be related with $\Gamma_{1}^{(0)}$ by imposing

$$
\Gamma_{1}^{(0)}[i]=-\sqrt{ }-1 \delta \lambda_{i}
$$

Substituting (85) and (86) into (84) and denoting $\theta=\left(\gamma^{*} \delta \lambda\right)$ we obtain

$$
\delta \ddot{\lambda}_{i}+n_{i}^{2} \sum_{k=1}^{\infty}(-1)^{k+1} b_{k}^{(i)} \sin k \theta=0
$$

from which after multiplication by $\gamma_{i}^{*}$ and summation follows the equation of libration

$$
\ddot{\theta}+\sum_{k=1}^{\infty}(-1)^{k+1}\left(\sum_{i=1}^{N} \gamma_{i}^{*} n_{i}^{2} b_{k}^{(i)}\right) \sin k \theta=0 \text {. }
$$




\section{Conclusion}

Experience with constructing the first-order theory revealed the practical efficiency of the method. Yet it became evident that the immediate continuation of the work by calculating the terms of the second and higher orders would involve the serious technical difficulties. Iterative version proposed here presents a trial to avoid these difficulties. Realization of the method on modern computers seems to merit attention.

\section{References}

Broucke, R.: 1969, Celes. Mech. 1, 110.

Broucke, R. and Garthwaite, K.: 1969, Celes. Mech. 1, 271.

Brumberg, V. A.: 1970, in G. E. O. Giacaglia (ed.), Periodic Orbits, Stability and Resonances, D. Reidel, Dordrecht, The Netherlands, p. 410.

Brumberg, V. A. and Chapront, J.: 1974, Celes. Mech. 8, 335.

Brumberg, V. A. and Egorova, A. V.: 1971, Observations of Artificial Celestial Bodies 62, 42 (in Russian); erratum: Bull. Inst. Theor. Astron. 13 (1972) 336 (in Russian).

Cherniack, J. R.: 1973, Celes. Mech. 7, 107.

Ferraz-Mello, S. : 1966, Bull. Astron. (3) 1, 287.

Jefferys, W. H.: 1970, Celes. Mech. 2, 474.

Jefferys, W. H.: 1972, Celes. Mech. 6, 117.

Kovalevsky, J.: 1971, Calculs de mécanique céleste sur ordinateur, São José dos Campos, Brazil.

Krasinsky, G. A. and Pius, L. Yu.: 1971, Observations of Artificial Celestial Bodies 62, 93 (in Russian). Marsden, B. G.: 1966, The Motions of the Galilean Satellites of Jupiter, Dissertation, Yale University.

Rom, A.: 1970, Celes. Mech. 1, 301.

Rom, A.: 1971, Celes. Mech. 3, 331.

Sagnier, J. L.: 1972, Séminaires du Bureau des Longitudes, 11 ème Année, No. 9.

Seidelmann, P. K.: 1970, Celes. Mech. 2, 134. 